www. revistadyo.com

\title{
Impacto del manejo del producto y prevención de la contaminación en la ventaja competitiva en negocios de mezcal de Oaxaca
}

\author{
Moisés González-Cruz, Patricia S. Sánchez-Medina, Juana Yolanda López Cruz \\ https://doi.org/10.37610/dyo.v0i75.606
}

Recibido: 19 de Octubre de 2020

Aceptado: 6 de Septiembre de 2021

\section{Resumen}

Este artículo analiza el impacto del manejo del producto y la prevención de la contaminación en la ventaja competitiva en costos, diferenciación y crecimiento empresarial, para ello se utiliza la visión basada en los recursos naturales (NRBV, por sus siglas en inglés), la cual fundamenta una relación entre diferentes estrategias ambientales y la ventaja competitiva. Se realizó una investigación cuantitativa, utilizado la técnica de mínimos cuadrados parciales para un modelo de ecuaciones estructurales (PLS-SEM, por sus siglas en inglés), basado en datos de encuestas realizadas a 61 negocios de mezcal ubicados en la "Región del Mezcal" del estado de Oaxaca. Los resultados demuestran que el manejo del producto impacta la ventaja competitiva en costos y el crecimiento empresarial, mientras que la prevención de la contaminación no arrojó resultados significativos, lo cual contradice los argumentos planteados en la NRBV. El artículo propone una serie de implicaciones teóricas y prácticas, enfatizando principalmente en la necesidad de proponer modelos más idóneos a contextos en subsistencia y la necesidad urgente de apoyo institucional al sector estudiado.

\section{Palabras clave}

Manejo del producto; Prevención de la contaminación; Ventaja competitiva; Visión basada en los recursos naturales; Negocios de mezcal.

\section{Introducción}

Actualmente la preocupación por la temática ambiental en las organizaciones ha ido en incremento, originado básicamente por la preocupación de las personas ante el rápido deterioro ambiental, ante esto las organizaciones se han visto en la necesidad de desarrollar procesos de producción que contribuyan al cuidado ambiental (SalgadoBeltrán, Gil, Subira y Beltrán-Morales, 2006; Salgado, 2019). A pesar de existir muchas investigaciones que han estudiado la influencia de distintas estrategias ambientales como el manejo del producto y la prevención de la contaminación en la ventaja competitiva de las empresas, los resultados encontrados son objeto de un debate en curso (AragónCorrea y Rubio-López, 2007). Según López-Gamero y Molina-Azorín (2016) la literatura teórica y empírica sobre la influencia de diferentes estrategias ambientales en la

Moisés González-Cruz (1)
mosheh_3003@hotmail.com
Tel.5170610 ext.82788, cel.9514190528
Patricia S. Sánchez-Medina (1)
ms_287506@yahoo.com.mx; psanchez@ipn.mx
Tel.5170610 ext.82788, cel.9511256446
iD ORCID:0000-0003-2949-3374
Juana Yolanda López Cruz (1)
jlopez_2000@yahoo.com.mx
iD ORCID:0000-0001-8812-2245

(1) Instituto Politécnico Nacional, CIIDIR - IPN Unidad Oaxaca. Hornos No. 1003, Col. Noche Buena, Santa Cruz Xoxocotlán, Oaxaca, C.P. 71230 ventaja competitiva de las empresas revela evidencias no concluyentes e incluso contradictorias. Existen estudios que consideran que diferentes estrategias ambientales (dentro de las que se encuentran el manejo del producto y la prevención de la contaminación) tienen impactos positivos en la ventaja competitiva de las empresas (Claver-Cortés y Molina-Azorín, 2009; Galdeano-Gómez, Céspedes-Lorente y Martínez-del Rio, 2008; González-Benito y GonzálezBenito, 2005; López-Gamero, Sharma y Vredenburg, 1998; Wahba, 2008), mientras que otras investigaciones no han encontrado esta relación o han encontrado una relación negativa (Hull y Rothenberg, 2008; Jaffe, Peterson, Portney y Stavings, 1995; Link y Naveh, 2006). El argumento central de la contradicción en los resultados radica principalmente en la idea de que la protección ambiental se asocia con costos adicionales impuestos por el gobierno, lo cual perjudica la competitividad (Ambec y Lanoie, 2008), aunque esta visión tiene su contraparte con Porter (1991) y más recientemente con Ramanathan, He, Black, Ghobadian y Gallear (2017) y Sánchez-Medina, Díaz-Pichardo, Bautista-Cruz y ToledoLópez (2015), respecto a que hay muchas formas de mejorar los aspectos ambientales de una empresa para conducir a una ventaja competitiva, sin que necesariamente aumenten los costos, esto conlleva a poca claridad y recientemente se muestra en la literatura que el análisis respecto a las estrategias ambientales particularmente el manejo del producto y la prevención de la contaminación sobre la ventaja competitiva (Hermundsdottir y Aspelund, 2021) sigue siendo complejo y por tanto se da evidencia de la necesidad de seguir investigando respecto a estos planteamientos. 
Aunado a lo anterior se tiene que las investigaciones sobre el manejo del producto y la prevención de la contaminación se han centrado mayormente en el impacto que las grandes empresas tienen sobre el medio ambiente (McKeiver y Gadenne, 2005). No obstante, se ha sugerido que el impacto colectivo de las Pequeñas y Medianas Empresas (PyMEs) sobre el medio ambiente es sustancial y podría compensar el impacto ambiental combinado de las grandes empresas (Hillary, 2000). En este estudio, nos enfocamos en el contexto de pequeños negocios, específicamente en el sector del mezcal de Oaxaca, un sector poco estudiado en el aspecto ambiental y que forma parte del porcentaje de PyMEs en México, aunque no se tiene un dato exacto de estos negocios, según el Directorio Estadístico de Unidades Empresariales (DENUE, 2015) se estima que existen un total de 334 establecimientos en el sector del mezcal del estado de Oaxaca. Según datos del Consejo Regulador del Mezcal (CRM, 2018) el estado de Oaxaca es el principal productor de esta bebida a nivel nacional, en el año 2011 la producción representaba el $77.4 \%$ y para el año 2018 representó el $92.3 \%$ de la producción nacional.

Debido al incremento en la producción de mezcal, se ha observado que se generan grandes cantidades de residuos sólidos y líquidos en toda la cadena productiva de la bebida, la mayoría de estos desechos contaminan el medio ambiente, lo que ha incentivado la preocupación de muchos negocios por implementar actividades que mitiguen el daño ambiental, estas actividades han sido implementadas sin ser reguladas por alguna norma ambiental y son realizadas de manera voluntaria. Al ser el cuidado ambiental una acción de tipo voluntaria en el sector mezcalero, el impacto de la actividad hacia el ambiente natural es aún descontrolado, y existe mucha desinformación sobre las ventajas que puede traer el cuidado medioambiental en los negocios de mezcal, otros problemas que aquejan al sector son la excesiva demanda del maguey mezcalero Angustifolia Haw por productores de tequila, lo que ha provocado el desplazamiento de la tecnología tradicional y ha venido a acentuar el bajo perfil tecnológico que se presenta en este sector, propiciando en algunos casos la disminución y abandono de la actividad productiva (Antonio, Orozco y Terán, 2015:1291).

Por otra parte, habría que considerar también que la producción del mezcal impacta de diversas formas a las comunidades que se dedican a su elaboración, dando una oportunidad de emprendimiento al generar valor al producto, las familias mezcaleras dependen de esta actividad y se sienten orgullosas de su tradición, preparando a sus nuevas generaciones para emprender. Los negocios de mezcal de Oaxaca se han convertido en un motor de desarrollo económico y social, por lo que es de vital importancia estudiar alternativas que permitan la mejora y permanencia de esta actividad.
Básicamente, está investigación contribuye al campo de la gestión ambiental en tres aspectos:

1) Estudia la relación entre el manejo del producto y la prevención de la contaminación sobre la ventaja competitiva, con la finalidad de aportar elementos que esclarezcan la relación entre estas variables dada la contradicción de resultados que se muestran en la literatura.

2) La investigación se realiza en una economía emergente en negocios artesanales de mezcal, los cuales han sido poco estudiados desde el ámbito de los aspectos ambientales, se espera que el estudio de este sector permita identificar oportunidades de crecimiento para la permanencia y estabilidad de estos negocios.

3) La investigación aporta al estudio de la visión basada en los recursos naturales, a partir del análisis de dos estrategias ambientales, el manejo del producto y la prevención de la contaminación, con ello se espera que los hallazgos encontrados permiten fortalecer la NRBV desde el ámbito de la gestión ambiental. Adicionalmente, se estudia el crecimiento empresarial como una ventaja competitiva, que si bien es cierto es mencionado en el planteamiento de la NRBV por Hart (1995), aún quedan vacíos respecto a esta variable por la ausencia de estudios empíricos.

El resto del documento se estructura de la siguiente manera: la sección 2 muestra el fundamento teórico de la investigación, la sección 3 explica la metodología, la sección 4 detalla el análisis de los datos y los resultados obtenidos, la sección 5 presenta las discusiones de los resultados, y finalmente la sección 6 incluye las conclusiones, limitaciones $\mathrm{y}$ directrices para futuras investigaciones.

\section{Revisión de literatura}

\section{Visión basada en los recursos naturales}

La visión basada en los recursos naturales explica la ventaja competitiva de la empresa mediante su relación con el ambiente natural, a partir del análisis de estrategias ambientales o capacidades estratégicas como son: el manejo del producto y la prevención de la contaminación (Hart, 1995). Cada una de estas estrategias tiene diferentes fuerzas ambientales impulsoras, se basa en diferentes recursos clave y puede impactar de forma diferente la ventaja competitiva (Hart y Dowell, 2011).

El manejo o administración del producto tienen como fuerza ambiental impulsora minimizar los costos en el ciclo de vida del producto, por lo que se incluye toda la cadena de valor del producto (Hart, 1995; Hart y Dowell, 2011). Las 
preocupaciones ambientales en esta estrategia se integran en cada etapa del ciclo de vida o cadena de valor del producto, es decir, desde la adquisición de las materias primas hasta el uso del producto, y obedece a las perspectivas externas de los stakeholders ante el requerimiento de aspectos ambientales en los procesos de diseño y desarrollo de productos, de ahí que la integración de los stakeholders sea un recurso clave para el desarrollo de esta estrategia (García-Román, SánchezMedina, Díaz-Pichardo y Caballero-Caballero, 2018; Hart, 1995; Kahupi, Eiríkur, Okorie y Millette, 2021). El costo ambiental del manejo del producto puede reducirse mediante la maximización en el uso de materiales no renovables, evitar el uso de materiales tóxicos, uso de materiales de bajo impacto ambiental en la elaboración del producto y la reutilización o reciclado al final de la vida útil de producto (García et al., 2018; Hart, 1995; Shrivastava, 1995).

Un buen manejo del producto, que incluya el uso de mejores prácticas de gestión ambiental conlleva a una ventaja competitiva, la cual se puede asegurar mediante una preferencia que se da principalmente por aspectos de diferenciación respecto a los competidores, la ventaja en diferenciación se enfoca en las características del producto y el mercado del producto, estos aspectos pueden incluir: el rediseño, envasado, producir de forma más responsable con el medio ambiente y desarrollar nuevos productos amigables con el medio ambiente (Albertini, 2013; Andersén, 2021; Sumrin, Gupta, Asaad, Wang, Bhattacharya y Foroudi, 2021).

En el contexto de los negocios de mezcal, Vázquez (2008) menciona que en el envasado del producto se utilizan botellas, tapas, sellos y cajas. En este sentido y siguiendo a Albertini (2013) y Sumrin et al., (2021), respecto a que algunos aspectos que incluye el manejo del producto son el envasado y empaquetado, en este trabajo se identifican como prácticas ambientales en el manejo del producto, aquellas relacionadas con el empaquetado como son la reutilización de materiales como cajas de cartón y embalaje para la comercialización de la bebida, y aspectos relacionados con el envasado como son el uso y reutilización de botellas de vidrio, de tal manera que se elimine el uso del plástico.

A diferencia del manejo del producto que se enfoca en el ciclo de vida o cadena de valor del producto, la prevención de la contaminación se orienta en una capacidad nueva construida en el proceso producción y en las operaciones de la empresa, esta estrategia tiene como fuerza ambiental impulsora minimizar las emisiones, afluentes y desperdicios y su recurso clave es la mejora continua (Hart, 1995), busca evitar la generación de residuos y emisiones en lugar de corregirlos, lo cual de acuerdo con Hart y Ahuja (1996) resulta más barato. A partir de la prevención de la contaminación las empresas pueden obtener ahorros significativos, lo que resulta en una ventaja competitiva en costos en comparación con sus competidores (Hart, 1995; Hart y Dowell, 2011), por lo que una ventaja de la empresa en costos puede obtenerse fácilmente con la implementación de una serie de medidas de prevención de la contaminación como lo plantea Schmidheiny, (1992), y se demuestra empíricamente en Meza y Juárez (2016).

Por tanto, eliminar los contaminantes del proceso de producción, permite aumentar la eficiencia al reducir los insumos demandados y disminuir consecuentemente los costos de la empresa, por ejemplo, se puede reducir el consumo de energía, agua, insumos, materiales, entre otros (Carter, Kale y Grimm, 2000; Christmann, 2000; Hart y Milstein, 2003; Mishra y Yadav, 2021).

En el contexto de los negocios de mezcal, Vázquez (2008) identificó las acciones que realizan los productores de esta bebida para minimizar los desechos generados en su producción. Por lo tanto, se identifican como prácticas encaminadas a prevenir la contaminación en los negocios de mezcal, la gestión de residuos sólidos y líquidos, consumo de agua y energía o algún otro combustible, aspectos que pueden ser medidos como prevención de la contaminación, ya que de acuerdo con Albertini (2013), la prevención de la contaminación comprende prácticas que reducen o eliminan la creación de contaminantes a través de un aumento eficiente en tres aspectos principalmente: uso de materias primas, energía y agua, lo cual es afín con lo que Hart y Ahuja (1996) argumentaron, respecto a que menos desperdicios significa una mejor utilización de los insumos, lo cual resulta en menores costos por materias primas y desechos (Hart y Ahuja, 1996).

Finalmente, cabe destacar que la implementación de acciones ambientales orientadas al manejo del producto $\mathrm{y}$ la prevención de la contaminación en las empresas permite eliminar los procesos de producción ambientalmente peligrosos, rediseñar los sistemas de productos existentes para reducir su impacto en el ciclo de vida y desarrollar productos con costos más bajos (Fraj-Andrés, MartínezSalinas y Matute-Vallejo, 2009).

\section{Manejo del producto y ventaja competitiva}

En el análisis del manejo del producto se pretende integrar las perspectivas de los distintos stakehoders, tanto en el diseño del producto como en el desarrollo de procesos (Hart, 1995). La intención con el estudio del manejo del producto es diseñar productos verdes que puedan colocarse en un mercado, por lo que las empresas deben diseñar una estrategia a través de toda su cadena de valor, proporcionando evidencia empírica de los efectos de esto sobre su ventaja competitiva (Yunus y Michalisin, 2016).

Ante esto, diversos trabajos se han realizado en relación con el estudio del manejo del producto y su impacto sobre la ventaja competitiva (Grimstad y Burgess, 2014; Hamdoun y Zouaoui, 2017; Liao, 2016; Magendans, 2015; Singjai, Winata y Kummer; 2018; Verma y Sangle, 2018; Yunus y Michalisin, 2016; Zameer, Wang y Yasmeen, 2020). 
Grimstad y Burgess (2014), realizan un estudio en pequeñas empresas dedicadas a la producción del vino en Australia, utilizando el modelo de la NRBV estos autores analizan la ventaja competitiva a partir del manejo del producto, enfocándose principalmente en la introducción de nuevos estándares para productos, Grimstad y Burgess (2014) encontraron una relación débil entre estas dos variables, es decir, las pequeñas empresas tienen menos ventaja competitiva para seguir estrategias encaminadas al manejo del producto. Esto derivado de las pocas acciones relacionadas con el manejo del producto en el sector del vino.

En un estudio realizado por Singjai et al., (2018), sobre las iniciativas verdes y la ventaja competitiva en la industria hotelera en países en desarrollo, empleando la NRBV encontraron que las iniciativas verdes, enfocadas al manejo del producto en cuanto a la interacción y comunicación con stakeholders, impacta significativamente la ventaja competitiva analizada a partir de los costos y diferenciación.

Magendans (2015), estudia la relación entre el manejo del producto y la ventaja competitiva apoyándose en la NRBV, a partir de estudios de caso en organizaciones manufactureras holandesas, encontrando que aun cuando las empresas analizadas no han logrado del todo acciones orientadas al manejo del producto, el desarrollo de capacidades hacia el manejo del producto resulta en una ventaja en reputación, pero no conduce a una ventaja en diferenciación como los sostiene Hart (1995).

Yunus y Michalisin (2016), apoyándose de los supuestos de la NRBV, estudian el manejo del producto a partir del análisis en la administración de la cadena de proveedores verdes, encontrando que la adopción de acciones en la cadena de suministro orientadas al manejo del producto contribuyen a una ventaja competitiva sostenida, estas estrategias ambientales requieren diversos grados de integración con los socios en la cadena de suministro de las empresas, mejorando su intercambio de conocimientos, el desarrollo de competencias y sus habilidades de coordinación.

De acuerdo con Verma y Sangle (2018), la implementación de la estrategia relacionada con el manejo del producto crea una ventaja de diferenciación para una empresa, esto basado en un análisis empírico de 60 empresas indias. Estas empresas colaboran con socios en la cadena de suministro para diseñar productos ecoeficientes y promover su transformación hacia la sostenibilidad con campañas de marketing y publicidad.

En un trabajo realizado por Zameer et al., (2020) en empresas manufactureras de China, se encontró que la imagen del producto ecológico, diseñado particularmente a partir de una marca ecológica contribuye significativamente a una competitividad ecológica, para ello el papel de los clientes es muy importante porque influyen en las empresas para que adopten una producción verde y con ello se refuerza la ventaja competitiva.
Otros trabajos que se han enfocado al manejo del producto, específicamente relacionados con cambios ambientales en el producto y que tienen que ver con la innovación, es el desarrollado por Liao (2016), quien encontró que la innovación ambiental del producto tiene un efecto positivo sobre la ventaja competitiva en diferenciación. Asimismo, Hamdoun y Zouaoui (2017) demostraron que las empresas ambientalmente responsables ofrecen productos ecológicos que crean una ventaja competitiva basada en la diferenciación,

A partir de la revisión de la literatura se observa que el manejo del producto determina una posición favorable de la empresa permitiéndole obtener una ventaja competitiva, sin embargo, como se puede apreciar a partir del análisis de los autores citados, esta ventaja competitiva no siempre está relacionada con una "diferenciación" ante los competidores como lo argumenta Hart (1995) en el planteamiento de la NRBV, por ello y dado que los negocios de mezcal, son pequeños, ubicados en contextos de subsistencia cuya venta es mayormente local y nacional, se espera que el manejo del producto en estos negocios conlleve a un impacto sobre la ventaja competitiva, sin importar que se trate únicamente en el aspecto de diferenciación como lo hace notar Magendans (2015).

Cabe señalar también que, en el sector de los negocios de mezcal, las acciones encaminadas al manejo del producto están principalmente relacionadas con el diseño del producto como son la reutilización de envases y el uso de tapone/ tapas de menor impacto ambiental y aquellas orientadas a la selección de proveedores verdes, estas acciones se han dado a partir de la influencia de stakeholders, principalmente clientes y asociaciones dedicadas a este sector. Como resultado de lo anterior, se establece la siguiente hipótesis.

H1: El manejo del producto impacta positivamente la ventaja competitiva en negocios de mezcal de Oaxaca.

\section{Prevención de la contaminación y ventaja competitiva}

La finalidad con la prevención de la contaminación es diseñar procesos verdes los cuales requerirán de mejora continua, para ello las empresas deben diseñar estrategias que les permitan incorporar aspectos verdes en sus procesos de producción, para el caso de empresas pequeñas en contextos de subsistencia, resulta interesante la incorporación de esta estrategia sin la necesidad de grandes inversiones, asimismo proporcionar evidencia empírica de los efectos de estas acciones sobre su ventaja competitiva.

A partir de lo anterior, se han desarrollado diversos trabajos que explican la ventaja competitiva con base en la implementación de acciones encaminadas a la prevención de la contaminación (Aboelmaged, 2018; Grimstad y Burgess, 2014; Liao, 2016; Meza y Juárez, 2016; Mishra y Yadav, 2021; Pereira-Moliner, Font, Tarí, Molina-Azorin, LópezGamero y Pertusa-Ortega, 2015; Verma y Sangle, 2016). 
Verma y Sangle (2016), estudian empresas de la India conocidas por su orientación hacia la sostenibilidad, y encuentran que las empresas prestan mayor atención a la gestión de residuos como una acción de prevención de la contaminación, básicamente porque esto les trae una ventaja competitiva respecto a sus costos iniciales, por lo que prefieren modificaciones de procesos menos propensas a la inversión. Otro estudio más reciente realizado también en empresas de la India por Mishra y Yadav (2021), utilizan la NBRV para analizar aspectos de prevención de la contaminación como es la innovación continua, encontrando que esta contribuye a la explicación de una ventaja competitiva en estas empresas.

Pereira-Moliner et al., (2015), estudiaron en el sector hotelero de España diversas prácticas orientadas a la prevención de la contaminación, como son: reducción en el consumo de agua, energía y recursos, así como el reúso y reciclaje de materiales, encontrando que la implementación voluntaria de estas acciones contribuye a una ventaja competitiva en costos, de igual manera Liao (2016), a partir de un estudio realizado en empresas manufactureras de China, estudia la reducción de desechos en el proceso de producción como una estrategia de prevención de la contaminación, encontrando que la implementación de esta acción tiene un efecto positivo en la ventaja competitiva en costos.

En un estudio realizado en el área Metropolitana de la Ciudad de México, Meza y Juárez (2016), analizan aspectos de prevención en el suministro de agua como una acción de producción más limpia, estos autores argumentan que en un largo plazo realizar acciones que garanticen un buen suministro del agua contribuirá a un escenario económico alentador.

Grimstad y Burgess (2014), en un estudio desarrollado en la industria del turismo del vino en Australia a partir del modelo de la NRBV, estos autores hallaron que las empresas que adoptan medidas respecto a la prevención de la contaminación como son reducción del agua, energía y recolección de desperdicio, tienen una ventaja respecto a la reducción en costos.

Mass, Schuster y Hartmann (2014), basados en la NRBV realizan una investigación en empresas de la industria de la logística, encontrando que la prevención de la contaminación ayuda a los proveedores de logística a lograr una ventaja competitiva en diferenciación.

D'Souza y Taghian (2018), analizaron distintas iniciativas ambientales (reducción de desechos, recuperación de recursos, y gestión y entendimiento de impactos ambientales de los productos químicos utilizados en la producción) encaminadas a la prevención de la contaminación y su influencia en la ventaja competitiva en medianas y pequeñas empresas de Arabia Saudita, sus resultados indican que las PyMEs que siguen estas iniciativas ambientales logran una ventaja competitiva porque reducen sus costos.
Aboelmaged (2018), realizó una investigación en empresas de Egipto, respecto a prácticas de manufactura sostenibles y su impacto en las capacidades competitivas, encontrando que la reducción de energía, uso de materiales, emisiones, aguas residuales, residuos sólidos y mejora en el uso de agua como prácticas de manufactura sostenibles y orientadas a la prevención de la contaminación impactan positiva y significativamente las capacidades competitivas de PyMes en Egipto.

Hermundsdottir y Aspelund (2021), realizan una revisión profunda en 100 publicaciones relevantes que analizan la innovación de sostenibilidad y la competitividad de la empresa, básicamente estos autores se enfocan en la innovación del proceso, estudiando aspectos como reducción de emisiones, desperdicios, energías y materiales de consumo, recursos, agua, gas, entre otros, aspectos relacionados con la prevención de la contaminación, Hermundsdottir y Aspelund (2021) encuentran que mayormente es reportado que la implementación de acciones respecto a la innovación de sostenibilidad conlleva a resultados competitivos como incremento en la creación de valor, reducción en costos y activos-no financieros.

Con base en los estudios descritos con anterioridad, la prevención de la contaminación impacta positivamente la ventaja competitiva de las empresas, en estos estudios se observa que esta ventaja competitiva está orientada mayormente a una reducción en costos, independientemente del sector de estudio porque al llevar a cabo prácticas orientadas a la prevención de la contaminación, las empresas pueden eliminar contaminantes de los procesos de producción, lo cual puede reducir el uso de insumos como la energía, agua y materiales, con ello se reducen los costos y por tanto la empresa puede tener una ventaja competitiva en costos en comparación con sus competidores. La producción de mezcal en Oaxaca ha cobrado gran importancia económica en los últimos años $\mathrm{y}$, ante la preocupación por los problemas ambientales actuales, existen productores de mezcal consientes con el daño ambiental que puede provocar su actividad, de ahí que lleven a cabo acciones encaminadas a la prevención de la contaminación como son reducción en el consumo de energía y tratamiento de residuos, con ello el artesano busca contribuir al cuidado del entorno natural pero también obtener una ventaja no necesariamente desde el punto de vista de la reducción en costos sino la oportunidad de colocarse bien en el mercado y generar mejores ganancias, como lo plantea Albertini (2003) en el sentido de que las acciones ambientales pueden generar un beneficio que le permita a la empresa no solo reducir costos sino maximizar sus utilidades.

El argumento anterior puede conducir a la siguiente hipótesis:

H2: La prevención de la contaminación impacta positivamente la ventaja competitiva en negocios de mezcal de Oaxaca. 


\section{Modelo de investigación}

El modelo de investigación propuesto para los negocios de mezcal de Oaxaca supone que la ventaja competitiva en costos, diferenciación y crecimiento empresarial se explica por el manejo del producto y la prevención de la contaminación (figura 1).
Figura 1 Modelo de

investigación.

Fuente: Elaboración propia

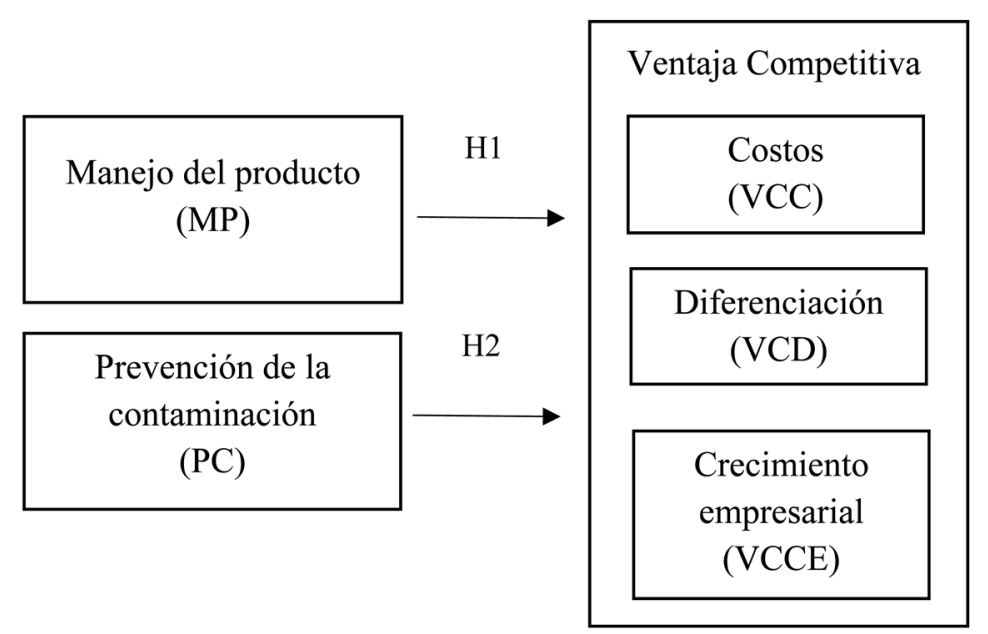

\section{Metodología}

En esta sección se explica el enfoque metodológico adoptado para el análisis empírico, con la finalidad de probar el modelo de investigación propuesto. Las siguientes secciones describen a detalle la muestra, así como las variables y sus medidas utilizadas.

\section{Población y Muestra}

La población objeto de estudio son los negocios de mezcal ubicados en la llamada "Región del Mezcal" del estado de Oaxaca, la cual de acuerdo con Antonio y Ramírez (2008) está ubicada en los Valles Centrales del estado, constituida por siete distritos políticos: Tlacolula, Yautepec, Miahuatlán, Ejutla, Ocotlán, Zimatlán y Sola de Vega, por las características agroclimáticas de esta región favorecen la producción de agave.

De acuerdo con el DENUE (2015), en el estado de Oaxaca hay 334 negocios de mezcal, incluyendo, almacenes, bodegas, expendios y fábricas de mezcal, sin embargo, no se cuenta con un número exacto de negocios ubicados en la región del mezcal, por tanto, la selección de la muestra se determinó con base en los siguientes criterios:
1) Negocios productores y comercializadores de mezcal, que llevarán a cabo toda la cadena de valor del mezcal, desde la adquisición de la materia prima, la producción, envasado y comercialización.

2) Negocios ubicados dentro de la Región del Mezcal.

3) Negocios de mezcal artesanal y ancestral.

4) Negocios de mezcal que implementarán acciones relacionadas con el cuidado ambiental (reutilización del bagazo y vinazas, reducción del consumo de agua, reutilización de cajas, envases y selección de materiales de bajo impacto ambiental para la comercialización de la bebida, entre otras).

5) Negocios de mezcal dispuestos a participar y proporcionar información para la investigación.

La muestra fue seleccionada de manera arbitraria, siguiendo los criterios descritos anteriormente, para la muestra mínima requerida se calculó el número de predictores, en este caso dos, con un nivel de significancia de 0.10 , un nivel de efecto medio y un poder estadístico de 0.8 , finalmente el tamaño requerido con estos datos y de acuerdo con Nitzl (2016) es de 54 unidades. En esta investigación se obtuvo un total de 61 negocios productores y comercializadores de mezcal, distribuidos como se muestra en la tabla 1 . 
Tabla 1 Distritos

entrevistados

Fuente: Elaboración propia

\begin{tabular}{|c|c|}
\hline Distrito & Entrevistados \\
\hline Tlacolula & 45 \\
\hline Ocotlán & 11 \\
\hline Sola de vega & 4 \\
\hline Miahuatlán & 1 \\
\hline Total & 61 \\
\hline
\end{tabular}

\section{Medición de las variables}

Para medir el manejo del producto y la prevención de la contaminación, a los encuestados se les preguntó sobre la frecuencia con la que realizaban acciones relacionadas con el manejo del producto (por ejemplo, selección de proveedores de envases ambientalmente responsables, uso de botellas de vidrio reciclado, entre otras) y la prevención de la contaminación (por ejemplo, eliminación, tratamiento/ almacenamiento de residuos y reducción de energía). Para medir esta variable sirvieron de guía los trabajos realizados por Carmona-Moreno, Céspedes-Lorente y Burgos-Jímenez (2004), de Yta (2015), González y González (2005) y McKeiver y Gadenne (2005), la información fue adaptada al contexto de los negocios de mezcal. Las respuestas fueron proporcionadas con base en una escala Likert, que va de $1=$ "nunca" a 5 = "siempre".

La ventaja competitiva se refiere a "la ventaja resultante de la implementación de actividades relacionadas con el cuidado del medio ambiente" (Christmann, 2000, p.24). Esta variable se midió considerando tres dimensiones: ventaja competitiva en costos, la cual hace referencia a una posición favorable que el negocio obtiene por la implementación de acciones ambientales, la ventaja competitiva en diferenciación supone una mejora de la imagen del negocio, así como la retención y atracción de nuevos clientes amigables con el medio ambiente, el crecimiento empresarial hace referencia al incremento de las utilidades, el volumen de producción y la adquisición de nuevos recursos. A los encuestados se les preguntó sobre su grado de acuerdo o desacuerdo en relación con una serie de resultados obtenidos por el negocio a partir de la implementación de acciones encaminadas al manejo del producto y prevención de la contaminación, las respuestas fueron proporcionadas con base en una escala Likert, que va de $1=$ "totalmente en desacuerdo" a 5 = "totalmente de acuerdo". La información para la medición de esta variable fue obtenida de Blázquez, Dorta y Verona (2006), Cater y Cater (2009), Christmann (2000) y López-Gamero et al., (2009), y adaptada al contexto de los negocios de mezcal.

Cabe señalar que estas variables fueron estudiadas porque son acciones que los artesanos dueños de negocios de mezcal realizan, asimismo, los ítems para medir cada una de estas variables y los cuales se detallan en la tabla 2, fueron seleccionados con base en una revisión exhaustiva de la literatura, pero también con base en su aplicación en el sector estudiado. 
Tabla 2 Constructos de la investigación Fuente: Elaboración propia

\begin{tabular}{|c|c|}
\hline \multicolumn{2}{|c|}{ Manejo del producto } \\
\hline MP15 & Toma en cuenta criterios ambientales en la selección de proveedores de envases \\
\hline MP18 & Utiliza envases o botellas de vidrio reciclado \\
\hline MP22 & $\begin{array}{l}\text { Elimina/trata/almacena de manera responsable el vidrio de los envases o } \\
\text { botellas defectuosas }\end{array}$ \\
\hline MP24 & Utiliza tapones o tapas de menor impacto ambiental \\
\hline \multicolumn{2}{|c|}{ Prevención de la contaminación } \\
\hline PC3 & Elimina/trata/almacena de forma responsable los residuos de bagazo \\
\hline PC6 & $\begin{array}{l}\text { Elimina/trata/almacena de manera ecológica las vinazas generadas por la } \\
\text { destilación del mezcal }\end{array}$ \\
\hline PC10 & Reducción el consumo de energía eléctrica \\
\hline \multicolumn{2}{|c|}{ Ventaja competitiva } \\
\hline \multicolumn{2}{|c|}{ Ventaja competitiva en costos } \\
\hline VCC35 & Nuestros costos por unidad de producto son más bajos \\
\hline VCC38 & $\begin{array}{l}\text { Bajos costos asociados con el reciclaje y reutilización de materiales y/o } \\
\text { residuos }\end{array}$ \\
\hline VCC39 & Menores costos asociados con la reducción del consumo de energía \\
\hline VCC40 & $\begin{array}{l}\text { En general nuestras acciones para abordar el tema ambiental mejoran nuestra } \\
\text { posición en costos en relación con nuestros competidores }\end{array}$ \\
\hline \multicolumn{2}{|c|}{ Ventaja competitiva en crecimiento } \\
\hline VCCE50 & Aumento en las utilidades \\
\hline VCCE52 & Aumento en el número de empleados \\
\hline VCCE53 & Aumento de la inversión fija \\
\hline \multicolumn{2}{|c|}{ Ventaja competitiva en diferenciación } \\
\hline VCD45 & Aumento de la calidad del producto \\
\hline VCD46 & Rapidez para satisfacer la demanda de los clientes \\
\hline VCD47 & Flexibilidad para satisfacer la demanda de los clientes \\
\hline
\end{tabular}

\section{Análisis de datos y resultados}

Para el análisis de los datos se aplicó la técnica de mínimos cuadrados parciales para un modelo de ecuaciones estructurales (PLS-SEM, por sus siglas en inglés), para ello se utilizó el software Smart PLS 3.0, los datos fueron analizados a través de dos fases que implican evaluar el modelo de medición y probar el modelo estructural.

\section{Modelo de medición}

Dos aspectos por considerar en el uso del PLS-SEM es que es menos sensible al tamaño de la muestra por lo que de acuerdo con Henseler y Sarstedt (2013), los datos de la muestra normal multivariante no son estrictamente necesarios y los resultados de los valores de curtosis y asimetría del modelo de medición están entre \pm 1 , lo que indica que no se han violado los supuestos de normalidad de los datos de la muestra (Aboelmaged, 2018).

La tabla 3 muestra los parámetros asociados con la evaluación del modelo de medición, como los valores de las cargas factoriales son superiores a 0.7 (Hair, Sarstedt, Hopkins y Kuppelwieser, 2014) y los valores alfa de Cronbach y valores de fiabilidad compuesta (CR) son mayores que .709, indica una confiabilidad interna aceptable de todas las variables (Aboelmaged, 2018). Asimismo, como la varianza media extraída (AVE) supera el valor requerido de 0.5 (Henseler, Hubona y Ray, 2016), se revela un adecuado nivel de validez convergente. 
Tabla 3 Validez y fiabilidad de los constructos.

Fuente: Elaboración propia

\begin{tabular}{|c|c|c|c|c|}
\hline Constructos de primer orden e ítems & Cargas & CR & AVE & $\begin{array}{c}\text { Alfa de } \\
\text { Cronbach }\end{array}$ \\
\hline Manejo del producto & & 0.925 & 0.756 & 0.891 \\
\hline MP15 & 0.912 & & & \\
\hline MP18 & 0.920 & & & \\
\hline MP22 & 0.795 & & & \\
\hline MP24 & 0.844 & & & \\
\hline Prevención de la contaminación & & 0.869 & 0.690 & 0.788 \\
\hline PC3 & 0.767 & & & \\
\hline PC6 & 0.862 & & & \\
\hline PC10 & 0.859 & & & \\
\hline Ventaja competitive en costos & & 0.971 & 0.895 & 0.961 \\
\hline VCC35 & 0.937 & & & \\
\hline VCC38 & 0.965 & & & \\
\hline VCC39 & 0.961 & & & \\
\hline VCC40 & 0.921 & & & \\
\hline Ventaja competitiva en crecimiento & & 0.984 & 0.952 & 0.975 \\
\hline VCCE50 & 0.962 & & & \\
\hline VCCE52 & 0.984 & & & \\
\hline VCCE53 & 0.982 & & & \\
\hline Ventaja competitive en diferenciación & & 0.933 & 0.823 & 0.902 \\
\hline VCD45 & 0.922 & & & \\
\hline VCD46 & 0.867 & & & \\
\hline VCD47 & 0.931 & & & \\
\hline
\end{tabular}

Para asegurar la validez discriminante, se siguió el criterio de Fornell y Larcker (1891), como se observa en la tabla 4, el valor de cada AVE en la diagonal es mayor que las correlaciones entre constructos, otra medida de validez discriminante utilizada son correlaciones de Heterotrait-
Monotrait (HTMT) con un valor de umbral menor a 0.90 (Henseler, Ringle y Sarstedt, 2015), como se observa en la tabla 4, el mayor índice de correlación HTMT es de .641, lo cual indica una validez discriminante aceptable y por tanto, un nivel de calidad satisfactorio de la medición.
Tabla 4 Discriminante (criterio de FornellLarcker). Fuente: Elaboración propia

\section{Procedimiento de Fornell y Larcker}

1. Manejo del producto

2. Prevención de la contaminación

3. Ventaja competitiva en costos

4. Ventaja competitiva en crecimiento

5. Ventaja competitiva en diferenciación

Heterotrait-monotrait (HTMT)
1. Manejo del producto
2. Prevención de la contaminación
3. Ventaja competitiva en costos
4. Ventaja competitiva en crecimiento
5. Ventaja competitiva en diferenciación

$\begin{array}{rrrrr}1 & 2 & 3 & 4 & 5 \\ .869 & & & & \\ .441 & .831 & & & \\ .516 & .354 & .946 & & \\ .599 & .396 & .447 & .976 & \\ .157 & .033 & .065 & .134 & \mathbf{. 9 0 7}\end{array}$

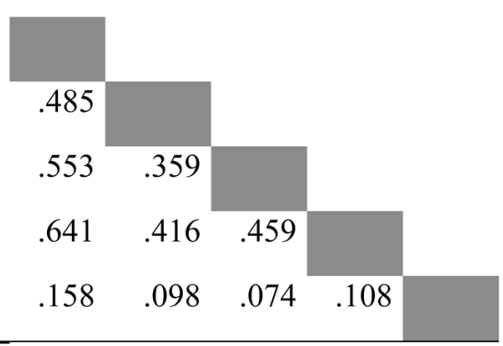




\section{Modelo estructural}

Se utilizó el método Bootstrap con 500 submuestras para probar la dirección, fuerza e importancia de los coeficientes path hipotetizados. Los índices considerados para el ajuste del modelo incluyen: $\mathrm{SRMR}=.078(<.08)$, dULS $=.941$ $(<.95), \quad \mathrm{dG}=.567 \quad(<.95)$, Chi-Square $=193.65 \mathrm{y}$ $\mathrm{NFI}=.820$, con base en esta información, se muestra un ajuste adecuado del modelo. Los límites sugeridos y colocados entre paréntesis son basados en Aboelmaged, (2018). Asimismo, Aboelmaged (2018) y Henseler y Sarstedt (2013), argumentan que los coeficientes path estandarizados y los valores R2 de las variables latentes endógenas, determinan la calidad del modelo estructural. Los coeficientes path y los valores R2 en esta investigación se muestran en la tabla 5 y figura 2.

La tabla 5 muestra el impacto del manejo del producto en la ventaja competitiva en $\operatorname{costos}(\beta=.447 ; \mathrm{p} \leq .001)$, y en la ventaja competitiva en crecimiento $(\beta=.527 ; p \leq .001)$, con lo cual la H1 es soportada. Respecto a la prevención de la contaminación, no se encontró ningún impacto significativo de esta variable sobre la ventaja competitiva en costos $(\beta=.157$; NS), en la ventaja competitiva en crecimiento $(\beta=.163$; NS), ni en la ventaja competitiva en diferenciación ( $\beta=-.045$; NS), por lo que este estudio no encontró elementos que respalden la H2.
Tabla 5 Coeficientes path. Fuente: Elaboración propia

Figura 2 Modelo Path. Fuente: Elaboración propia

\begin{tabular}{|l|l|c|c|}
\hline Relaciones hipóteticas & $\begin{array}{l}\text { Coeficientes } \\
\text { path } \\
\text { (Estandarizados } \\
\beta)\end{array}$ & $\begin{array}{l}\text { Estadistico t } \\
\text { student } \\
\text { (Bootstrapping) }\end{array}$ & Valor P \\
\hline MP $\longrightarrow$ VCC & .447 & 3.605 & .000 \\
\hline MP $\longrightarrow$ VCCE & .527 & 5.771 & .000 \\
\hline MP $\longrightarrow$ VCD & .176 & 1.165 & .245 \\
\hline PC $\longrightarrow$ VCC & .157 & 1.271 & .204 \\
\hline PC $\longrightarrow$ VCCE & .163 & 1.442 & .150 \\
\hline PC $\longrightarrow$ VCD & -.045 & 0.210 & .834 \\
\hline
\end{tabular}

Prácticas sostenibles

Ventaja competitiva

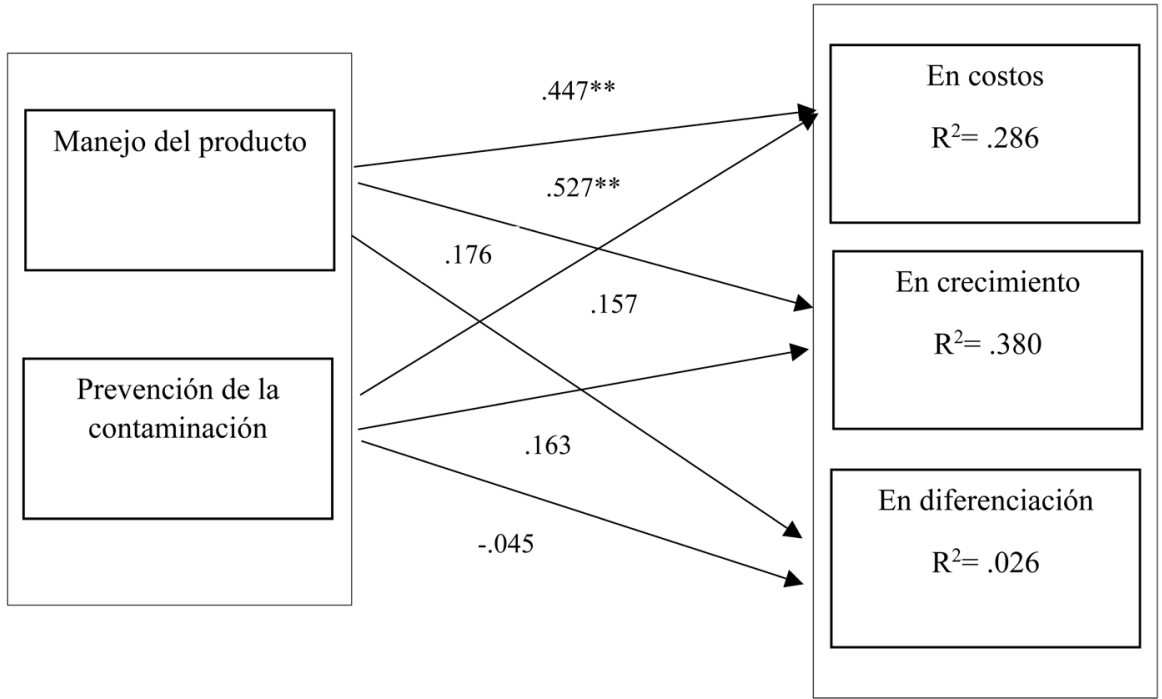




\section{Discusiones}

En este estudio se propone un modelo para explicar la ventaja competitiva a partir de acciones encaminadas al manejo del producto y prevención de la contaminación, dicho modelo se sustenta en la teoría NBRV y con fundamento en un análisis empírico a 61 negocios de mezcal de Oaxaca, se encontraron resultados importantes, los cuales apoyan parcialmente las hipótesis planteadas y resultan contrastantes cuando se analizan a la luz de la NBRV y de investigaciones anteriores.

Respecto al efecto del manejo del producto sobre la ventaja competitiva, el resultado obtenido muestra que las acciones encaminadas al manejo del producto impactan en la ventaja competitiva en costos y el crecimiento empresarial. Este hallazgo coincide con Singjai et al., (2018) quienes encontraron también que iniciativas verdes enfocadas al manejo del producto, en cuanto a la interacción de stakeholders impacta sobre la ventaja competitiva en costos, cabe resaltar que la investigación realizada por Singjai et al., (2018) se lleva a cabo también en el contexto de un país en vías de desarrollo, sin embargo, el resultado es inconsistente con Hamdoun y Zouaoui (2017), Liao (2016) y Verma y Sangle (2018), quienes encontraron que el manejo del producto explica una ventaja competitiva en diferenciación.

Una posible explicación a lo anterior es que en su mayoría los negocios de mezcal se encuentran agrupados en asociaciones como el Consejo Regulador del Mezcal, por medio de estos organismos reciben capacitación y orientación para el desarrollo de sus actividades empresariales, en muchas ocasiones lo hacen de manera conjunta, lo que probablemente obstaculiza que alcancen una ventaja competitiva en diferenciación respecto a sus competidores. Respecto a la ventaja competitiva en costos y crecimiento empresarial que los negocios de mezcal alcanzan, puede deberse a que, si bien están organizados en asociaciones, la inversión que realizan para implementar iniciativas ambientales orientadas al manejo del producto depende de cada negocio y de las exigencias de los stakeholders, con ello estos negocios buscan minimizar sus costos y les permite también un mejor crecimiento en comparación con otros negocios de mezcal.

Resulta importante mencionar que esta investigación apoya el argumento de Magendans (2015), en el sentido de que los resultados aquí obtenidos conducen a la explicación de la ventaja competitiva en costos y crecimiento empresarial pero no en diferenciación, como los sostiene Hart (1995) en el planteamiento de la NBRV, cuando se explica que la capacidad estratégica relacionada al manejo del producto permite tener una posición anticipada a los competidores a partir de aspectos de diferenciación. Probablemente esto dependerá del contexto analizado, ya que los hallazgos encontrados en este estudio como los de Singjai et al., (2018) son encontrados en el contexto de países en desarrollo.
Con respecto a si la prevención de la contaminación impacta en la ventaja competitiva, este estudio no encontró elementos que apoyen este supuesto, por lo que los resultados aquí encontrados contradicen los hallazgos de investigaciones previas como las de Aboelmaged (2018), D'Souza y Taghian (2018), Hermundsdottir y Aspelund (2021), Liao (2016) y Pereira-Moliner et al., (2015).

Al parecer las acciones realizadas por los negocios de mezcal, respecto a la prevención de la contaminación y que son orientadas básicamente al tratamiento de residuos y reducción de energía son aún incipientes para lograr que impacten en su ventaja competitiva, lo anterior probablemente se deba a que al artesano le resulta más barato orientarse en aspectos de su producto que en sus procesos.

Los resultados de esta investigación muestran claramente que es posible obtener beneficios competitivos como resultado de la implementación de actividades relacionadas con el cuidado ambiental, con este trabajo se demuestra que aun cuando los negocios de mezcal son pequeños han implementado actividades voluntarias para reducir su impacto ambiental, lo han realizado a partir de la exigencia de ciertos stakeholders, más que por una certificación ambiental formal.

Finalmente, mayor investigación se necesita respecto al planteamiento de la NBRV con relación al tipo de ventaja competitiva que puede explicarse a partir de la implementación de distintas capacidades estratégicas, ya que con este trabajo se expone que efectivamente el manejo del producto conduce a una ventaja competitiva pero no precisamente en diferenciación (Magendans, 2015) y la prevención de la contaminación no necesariamente conduce a una ventaja competitiva.

\section{Conclusiones}

Estudiar el manejo del producto y la prevención de la contaminación para explicar la ventaja competitiva a partir de la NBRV, aun cuando el tema ha sido ampliamente estudiado, sigue siendo de discusión para entender aspectos de gestión ambiental empresarial, en contraste con la literatura previa en torno a esta temática, este artículo hace un intento por explicar aspectos ambientales y sus beneficios en un contexto de negocios de mezcal en Oaxaca, México.

El estudio contribuye al cuerpo de literatura en gestión ambiental a partir de los siguientes aspectos: 1) se respalda el enfoque positivo existente en la literatura, con relación a que el cuidado ambiental conlleva a una ventaja competitiva sin que estén asociados en ello costos adicionales. Un aspecto fundamental para ello y de acuerdo con el contexto estudiado, es la voluntad e interés por parte de los artesanos de implementar prácticas ambientales, particularmente estas prácticas son básicas sin incluir grandes inversiones. 
2) Se estudio un contexto en subsistencia en un país en vías de desarrollo, el contexto de los negocios de mezcal regularmente ha sido muy poco analizado aun cuando la actividad es importante para el estado, por lo que este estudio contribuye al analizar este sector y encontrar que las iniciativas de los artesanos respecto al manejo de la bebida están impactando en sus costos y en el crecimiento del negocio, por tanto, se debe impulsar al sector a partir de financiamiento y capacitación para seguir realizando prácticas ambientales que les permita reforzar las ventajas competitivas que ya tienen y obtener otras ventajas para mantenerse en el mercado, más aún ante la competencia de otros productores de bebidas como el tequila en Guadalajara, por ejemplo. 3) Se contribuye al estudio de la NBRV, principalmente en dos vertientes: primero, se encontró que el manejo del producto conlleva a la explicación de una ventaja competitiva en costos y crecimiento, pero no en diferenciación como se argumenta en la NBRV, quizás esto se deba a que la investigación se realizó en un contexto de un país en vías de desarrollo, por tanto, mayor investigación es necesaria para incluir otras variables que puedan respaldar o contradecir los hallazgos aquí reportados. Segundo, se estudia el crecimiento empresarial como una ventaja competitiva, lo cual ha sido escasamente abordado dentro de la literatura y se encontró que el crecimiento empresarial en los negocios de mezcal se explica a partir del manejo ambiental que los artesanos hacen de su bebida, por tanto este estudio sienta las bases para una mayor investigación en torno al crecimiento empresarial como una ventaja competitiva a partir de diversas capacidades estratégicas como lo son el manejo del producto y la prevención de la contaminación. Lo anterior proporciona elementos relevantes a considerar con la finalidad de proponer modelos más idóneos a las necesidades de contextos en subsistencia.

Con este estudio se corrobora que aun cuando el sector de mezcal está formado en su mayoría por negocios pequeños en subsistencia, se pueden implementar diversas prácticas respetuosas con el medio ambiente, estas prácticas pueden mejorar la ventaja competitiva de estos negocios, lo que les permite tener una mejor posición respecto a sus competidores. Sin embargo, es importante recalcar que aun cuando los resultados encontrados en esta investigación son prometedores, queda mucho por trabajar en este tema, por lo que, el apoyo del gobierno y dependencias profesionales en materia de cuidado ambiental es muy necesario para impulsar la adopción de prácticas ambientales en el sector de mezcal.

Para los artesanos, dueños de los negocios de mezcal, esta investigación proporciona información relevante sobre las diferentes actividades relacionadas con el cuidado del medio ambiente, las cuales pueden seguir implementándose para reforzar los beneficios obtenidos en costos y crecimiento empresarial, pero también pueden implementarse otras prácticas ambientales relacionadas con la prevención de la contaminación con la finalidad de traer otros beneficios competitivos al sector y mantenerse en el mercado. Para el gobierno y las instituciones que se enfocan en temas de medio ambiente, los resultados indican la necesidad de proporcionar capacitación a los artesanos mezcaleros, con la finalidad de orientarlos en procesos sostenibles y explicarles las ventajas que podrían obtener si utilizan prácticas ambientales.

\section{Limitaciones y directrices para futuras investigaciones}

Si bien esta investigación tiene importantes contribuciones a la literatura en gestión ambiental sobre el efecto del manejo del producto y la prevención de la contaminación en la ventaja competitiva, presenta ciertas limitaciones: Primero, se sugiere que, para investigaciones futuras sobre aspectos ambientales y ventaja competitiva, se utilice una muestra más grande que permite validar mejor el estudio actual en diferentes entornos. Segundo, dado que en el contexto estudiado los artesanos son reconocidos por la reputación que ganan a través de los años de ser mezcaleros en su comunidad, para investigaciones futuras se recomienda estudiar aspectos de una ventaja competitiva en reputación como lo argumenta Magendans (2015). Tercero, mayores acciones realizadas con la prevención de la contaminación deberían identificarse y estudiarse en el sector mezcalero, con la intención de poder identificar si hay un cambio con los resultados aquí obtenidos. Cuarto, debido a la gran variedad en términos relacionados con el manejo del producto y la prevención de la contaminación, es posible que estudios relevantes que utilizan términos distintos a las palabras clave utilizadas fueron no encontrados en la literatura.

\section{Referencias}

ABOELMAGED, M. (2018). «The drivers of sustainable manufacturing practices in Egyptian SMEs and their impact on competitive capabilities: A PLS-SEM model». Journal of Cleaner Production, 175, pp. 207-221.

ALBERTINI, E. (2013). «Does environmental management improve financial performance? A meta-analytical review». Organization \& Environment, 26 (4), pp. 431457.

AMBEC, S., y LANOIE, P. (2008). «Does it pay to be green? A systematic overview». The Academy of Management Perspectives, 22 (4), pp. 45-62.

ANDERSÉN, J. (2021). «A relational natural-resourcebased view on product innovation: The influence of green product innovation and green suppliers on differentiation advantage in small manufacturing firms». Technovation, 104 https://doi.org/10.1016/j. technovation.2021.102254

ANTONIO, B.J., OROZCO, C.S., y TERÁN, M.E. (2015). «La disminución de la producción artesanal de mezcal en la Región del mezcal de Oaxaca, México». Revista Mexicana de Ciencias Agrícolas, 6 (6), pp. 1291-1305. 
ANTONIO, B.J., y RAMÍREZ, J. (2008). «Agricultura y pluriactividad de los pequeños productores de agave en la región del mezcal, Oaxaca, México». Agricultura Técnica en México, 34 (4), pp. 443-451.

ARAGÓN-CORREA, J. A., y RUBIO-LÓPEZ, E. A. (2007). «Proactive corporate environmental strategies: myths and misunderstandings». Long Range Planning, 40 (3), pp. 357-381.

BLÁZQUEZ S. F., DORTA, V. J., y VERONA, M. M. (2006). «Concepto, Perspectivas y Medida del Crecimiento Empresarial». Cuaderno Administrativo, 19 (31), pp. 165-195.

CARMONA-MORENO, E., CÉSPEDES-LORENTE, J., y BURGOS-JIMÉNEZ, J. (2004). «Environmental Strategy in Spanish Hotels: Contextual Factors and Performance». The Service Industries Journal, 24 (3), pp. 101-130.

CARTER, C. R., KALE, R. P., y GRIMM, C. M. (2000). «Environmental purchasing and firm performance: an empirical investigation». Transportation Research Part E: Logistics and Transportation Review, 36 (3), pp. 219228.

CATER, T., y CATER, B. (2009). «Intangible Resources as Antecedents of a Company's Competitive Advantage and Performance». Journal of East European Studies, 14 (2), pp. 186-209.

CHRISTMANN, P. (2000). «Effects of "Best Practices" of environmental management on cost advantage: The role of complementary assets». Academy of Management Journal, 43 (4), pp. 663-680.

CRM. (2018). «Informe Estadístico 2018 del Consejo Regulador del Mezcal». Consultado en:

http://www.crm.org.mx/PDF/INF_ACTIVIDADES/INFORME2018.pdf

DE YTA, C.D. (2015). «Capacidades Estratégicas Ambientales y Desempeño Empresarial en el Sector Hotelero Mexicano» (Tesis Doctoral). Instituto de Estudios Universitario A. C. Puebla, México.

DENUE. (2015). «Directorio Estadístico Nacional de Unidades Económicas». Consultado en: http://www. inegi.org.mx/app/mapa/denue/default.aspx

D'SOUZA, C. y TAGHIAN, M. (2018). «Small and medium size firm's marketing competitive advantage and environmental initiatives in the Middle East». Journal of Strategic Marketing, 26 (7), pp. 568-582.
FRAJ-ANDRÉS, E., MARTÍNEZ-SALINAS, E., y MATUTE-VALLEJO, J. (2009). «Factors affecting corporate environmental strategy in Spanish industrial firms». Business Strategy and the Environment, 18 (8), pp. 500-514.

FORNELL, C., y LARCKER, D. (1981). «Evaluating structural equation models with unobservable variables and measurement error». Journal of Marketing Research, 18 (1), pp. 39-50.

GALDEANO-GÓMEZ, E., CÉSPEDES-LORENTE，J., y MARTÍNEZ-DEL RIO, J. (2008). «Environmental performance and spillover effects on productivity: evidence from horticultural firms». Journal of Environmental Management, 88 (4), pp. 1552-1561.

GARCÍA-ROMÁN, I., SÁNCHEZ-MEDINA, P. S., DÍAZPICHARDO, R., y CABALLERO-CABALLERO, M. (2018). «Environmental strategy and competitive advantage in mescal businesses in Oaxaca, Mexico». International Journal of Management and Marketing, 11 (1), pp. 41-54.

GONZÁLEZ-BENITO, J., y GONZÁLEZ-BENITO, O. (2005). «Environmental proactivity and business performance: an empirical analysis». Omega, 33 (1), pp. $1-15$.

GRIMSTAD, S., y BURGESS, J. (2014). «Environmental sustainability and competitive advantage in a wine tourism micro-cluster». Management Research Review, 37 (6), pp. 553-573.

HAIR, J. F., SARSTEDT, M., HOPKINS, L., y KUPPELWIESER, V. G. (2014). «Partial least squares structural equation modeling (PLS-SEM) ». European Business Review, 26, pp. 106-121

HAMDOUN, M., y ZOUAOUI, M. (2017). «Impact of environmental management on competitive advantage of Tunisian companies: the mediator role of organizational culture». International Review of Management and Marketing, 7 (2), pp. 76-82.

HART, S. (1995). «A natural resourced-based view of the firm». The Academy of Management Review, 20 (4), pp. 986-1014.

HART, S. L., y MILSTEIN, M. B. (2003). «Creating sustainable value». Academy of Management Executive, 17 (2), pp. 56-69.

HART, S., y DOWELL, G. (2011). "A natural resourcebased view of the firm: fifteen years after». Journal of Management, 37 (5), pp. 1464-1479. 
HART, S., y AHUJA, G. (1996). «Does it pay to be green? An empirical examination of the relationship between emission reduction and firm performance». Business Strategy and the Environment, 5, pp. 30-37.

HENSELER, J., y SARSTEDT, M., (2013). «Goodnessof-fit indices for partial least squares path modeling». Computational Statistics, 28 (2), pp. 565-580.

HENSELER, J., HUBONA, G. S., y RAY, P. A. (2016). «Using PLS path modeling in new technology research: Updated guidelines». Industrial Management \& Data Systems, 116, pp. 1-19.

HENSELER, J., RINGLE, C. M., y SARSTEDT, M. (2015). «A new criterion for assessing discriminant validity in variance-based structural equation modeling». Journal of the Academy of Marketing Science, 43, pp. 115-135.

HERMUNDSDOTTIR, F., y ASPELUND, A. (2021). «Sustainability innovations and firm competitiveness: A review». Journal of Cleaner Production, 280 (1). https://doi.org/10.1016/j.jclepro.2020.124715

HILLARY, R. (2000). Small and medium-sized enterprises and the environment: business imperatives. Sheffield: Greenleaf Publishing.

HULL, C. E., y ROTHENBERG, S. (2008). «Firm performance: the interactions of corporate social performance with innovation and industry differentiation». Strategic Management Journal, 29 (7), pp. 781-789.

JAFFE, A. B., PETERSON, S. R., PORTNEY, P. R., y STAVINS, R. N. (1995). «Environmental regulation and the competitiveness of U.S. manufacturing: what does the evidence tell us? » Journal of Economic Literature, 33 (1), pp. 132-163.

KAHUPI, I., EIRÍKUR, H. C., OKORIE, O., y MILLETTE, S. (2021). «Building competitive advantage with sustainable products -A case study perspective of stakeholders». Journal of Cleaner Production, 289, https://doi.org/10.1016/j.jclepro.2020.125699

LIAO, Z. (2016). «Temporal cognition, environmental innovation and the competitive advantage of enterprises». Journal of Cleaner Production, 135, pp. 1045-1053.

LINK, S., y NAVEH, E. (2006). «Standardization and discretion: does the environmental standard ISO 14001 lead to performance benefits?» IEEE Transactions on Engineering Management, 53 (4), pp. 508-519.
LÓPEZ-GAMERO, M. D., CLAVER-CORTÉS, E., y MOLINA-AZORÍN, J. F. (2009). «The whole relationship between environmental variables and firm performance:

competitive advantage and firm resources as mediator variables». Journal of Environmental Management, 90 (10), pp. 10-21.

LÓPEZ-GAMERO, M. D., y MOLINA-AZORÍN, J. F. (2016). «Environmental management and firm competitiveness: the joint analysis of external and internal elements». Long Range Planning, 49, pp. 746763.

MAGENDANS, E. C. (2015). «The challenge of attaining product stewardship: Multiple case study of environmental capability development in Dutch manufacturing organizations» (Master Thesis). Newcastle University Business School and Faculty of Economic and Business at University of Groningen.

MASS, S., SCHUSTER, T., y HARTMANN, E. (2014). «Pollution prevention and service stewardship strategies in the third-party logistics industry: Effects on firm differentiation and the moderating role of environmental communication». Business Strategy and the Environment, 23 (1), pp. 38-55.

MCKEIVER, C., y GADENNE, D. (2005). «Environmental management systems in small and medium businesses». International Small Business Journal, 23 (5), pp. 513537.

MEZA, S.A., y JUÁREZ, N.M. (2016). «Cost-effective advantages due to clean technologies: water compliance scenarios for a Mexican paper mill». Journal of Cleaner Production, 112 (5), pp. 4701-4709.

MISHRA, P., y YADAV, M. (2021). «Environmental capabilities, proactive environmental strategy and competitive advantage: A natural-resource-based view of firms operating in India». Journal of Cleaner Production, 291. https://doi.org/10.1016/j.jclepro.2020.125249

NITZL, C. (2016). «The use of partial least squares structural equation modelling (PLS-SEM) in management accounting research: Directions for future theory development». Journal of Accounting Literature, 37, pp. 19-35.

PEREIRA-MOLINER, J., FONT, X., TARÍ, J.J., MOLINAAZORIN, J. F., LÓPEZ-GAMERO, M. D., y PERTUSA-ORTEGA, E. M. (2015). «The Holy Grail: environmental management, competitive advantage and business performance in the Spanish hotel industry». International Journal of Contemporary Hospitality, 27 (4), pp. 714-738. 
PORTER, M. (1991). America's Green Strategy. Scientific American.

RAMANATHAN, R., HE, Q., BLACK, A., GHOBADIAN, A., y GALLEAR, D. (2017). «Environmental regulations, innovation and firm performance: A revisit of the Porter hypothesis». Journal of Cleaner Production, 155 (2), pp. 79-92.

SALGADO-BELTRÁN, L., GIL, A., SUBIRA, E., y BELTRÁN-MORALES, L.F. (2006). «Teoría de efectos olvidados en el consumo sustentable de productos ecológicos». Pp. 223-239. En: Libro LUIS F. BELTRÁNMORALES, JOSÉ URCIAGA-GARCÍA y ALFREDO ORTEGA-RUBIO (Eds). Desarrollo Sustentable: ¿Mito o Realidad? CIBNOR, SC., CONACYT, SEMARNAT, INE, México.

SALGADO, L. (2019). «Segmentación de los consumidores de alimentos orgánicos según sus actitudes, valores y creencias ambientales». Contaduría y Administración, 64 (2), pp. 1-22.

SÁNCHEZ-MEDINA， P.S., DÍAZ-PICHARDO, R., BAUTISTA-CRUZ, A., y TOLEDO-LÓPEZ, A. (2015). «Environmental compliance and economic and environmental performance: Evidence from handicrafts small businesses in Mexico». Journal of Business Ethics, 126, pp. 381-393.

SCHMIDHEINY, S. (1992). Changing course. Cambridge: MIT Press.

SHARMA, S., y VREDENBURG, H. (1998). «Proactive corporate environmental strategy and the development of competitively valuable organizational capabilities». Strategic Management Journal, 19, pp. 729-753.

SHRIVASTAVA, P. (1995). «Environmental technologies and competitive advantage». Strategic Management Journal, 16 (S1), pp. 183-200.
SINGJAI, K., WINATA, L., y KUMMER, T. (2018). «Green initiatives and their competitive advantage for the hotel industry in developing countries». International Journal of Hospitality Management, 75, pp.131-143.

SUMRIN, S., GUPTA, S., ASAAD, Y., WANG, Y., BHATTACHARYA, S., y FOROUDI, P. (2021). «Ecoinnovation for environment and waste prevention». Journal of Business Research, 122, pp. 627-639. https://doi.org/10.1016/j.jbusres.2020.08.001

VÁZQUEZ, B. A. (2008). «Análisis de la eco-eficiencia en la producción de mezcal» (Tesis Maestría), Centro Interdisciplinario de Investigación para el Desarrollo Integral Regional, Instituto Politécnico Nacional.

VERMA, B.K., y SANGLE, S. (2018). «Product stewardship strategy: A study of Indian firms». Corporate Social Responsibility and Environment Management, 25(2), pp. 124-134.

VERMA, B.K., y SANGLE, S. (2016). «Pollution prevention strategy: A study of Indian firms». Journal of Cleaner Production, 133, pp. 795-802.

WAHBA, H. (2008). «Does the market value corporate environmental responsibility? An empirical examination». Corporate Social Responsibility and Environmental Management, 15 (2), pp. 89-99.

YUNUS, E.N., y MICHALISIN, M.D. (2016). «Sustained competitive advantage through green supply chain management practices: a natural-resource-based view approach». International Journal of Services and Operations Management, 25(2), pp. 135-154.

ZAMEER, H., WANG, Y., y YASMEEN, H. (2020). «Reinforcing green competitive advantage through green production, creativity and green brand image: Implications for cleaner production in China». Journal of Cleaner Production, 247, https://doi.org/10.1016/j. jclepro.2019.119119 